\title{
Integrated power supply system for station equipment of rail traffic control
}

\author{
Ryszard Mielnik ${ }^{1}$, and Zofia Wróbel ${ }^{2, *}$ \\ ${ }^{1}$ Cracow University of Technology, Institute of Electromechanical Energy Conversions E-2, ul. Warszawska 24, 31-155, Kraków, \\ Poland \\ ${ }^{2}$ PKP Polish Railway Lines JSC, Railway Lines Establishment in Rzeszów, ul. St. Batorego 24, 35-005, Rzeszów, Poland
}

\begin{abstract}
Railway Traffic Control Systems (RTCS) provide a safe, reliable and efficient movement of rolling stock on railway networks. RTCSs are classified as critical process systems. The power supply system is required to provide a high level of power reliability so that a station equipment of an RTCS safely executes the rail traffic process. The article describes typical solutions of power supply of station equipment of RTCSs. Analysis of these solutions led to the development of assumptions and structures for the integrated power supply system of station equipment. Useful tools for the formal modeling of such systems can be Petri Nets. These nets are a graphical and formal tool for modeling, formal analysis, and design of discrete event systems. The basic element of the integrated power system which is described in the article is the Automatic Reserve Switching system (ARS system). The ARS controller Marked Petri Net (ARS controller MPN) that has been developed by the authors is presented here. The net was subject to reduction resulting in an ARS controller hierarchical simplified MPN. The article also presents the practical application of the ARS controller built using the hardware platform and graphical programming environment LabView National Instruments (NI).
\end{abstract}

\section{Introduction}

Nowadays measurement and control digital systems (MCD systems) are commonly used in industrial processes [1]. These systems are built on the basis of hardware platforms such as PLC's (Programmable Logic Controller), industrial computers or embedded systems. The functionality of MCD systems depends on software applications as implemented by particular users. Thus, the quality of the action of this system is influenced not only by the hardware platform, but also by its user's software. The need to ensure high reliability of the software and the reliability of the entire MCD system is required especially in the field of the so-called critical applications. In these applications malfunction or failure of MCDs during their operation may lead to abnormal functioning of supervised process resulting in the loss of life and material waste. Ensuring the required quality of an MCD system during the design and prototyping process requires the use of appropriate tools and methods to formalize its functions and actions. Suitable instruments which can be used for this purpose are Petri Nets. They are graphic and formal tools for modeling, analysis and design of discrete event systems. The model represented by such nets allows for the analysis of the characteristics of the system's behavior, and its evaluation in each phase of the system's life cycle.

The aim of this publication is to present the opportunities for using Petri Nets in modeling MCD systems in critical applications $[2,3,4,5,6]$, i.e. the integrated controller of the Automatic Reserve Switching

\footnotetext{
* Corresponding author: zwrobel@prz.edu.pl
}

(ARS) device of the power supply system of Railway Traffic Control Systems (RTCS). Here, the ARS controller Marked Petri Net is presented. This publication also shows the practical implementation of such ARS controller. It has been implemented using the hardware platform of the family of controllers NI CompactRIO and a software application implemented in the National Instruments graphical environment LabView $[6,7,8]$.

\section{Power supply system for Railway Traffic Control Systems}

\subsection{Railway Traffic Control Systems}

Railway Traffic Control Systems (RTCS) provide safe, reliable and efficient means for the movement of rolling stock on railway networks [2]. Therefore, RTCSs are classified as critical equipment and systems $[4,5,6,7]$. The history of RTCSs is dated back to the end of the 19th century when the industrial revolution introduced rolling stock for the transport of goods and people. Initially, railway traffic safety devices were simple, and the main role in the decision making and execution process was in the hands of human operators. Currently, mounted station RTCS systems are relay and computer based systems or computer based systems only [2, 3]. In relay and computer based systems, the security is ensured in the relay layer, and the setting functions are performed by a computer control panel [2]. The 
development of information and computer technology has led to the construction of secure computer systems. For systems of this type, PLCs or computers with "2 of 2 " or "2 of 3 " voting systems are used to provide the required level of security $[2,3,7]$. The use of relay and computer based systems or computer based systems requires the provision of adequate reliability and quality of power supply [9]. Therefore, new, more suitable power supply systems are introduced so that they can meet strict sets of requirements.

\subsection{Power supply systems for RTCS}

The current requirements for the design and manufacture of power supply systems for RTCS systems are contained in Chapter 13 of Management Order No. 1/2014 of PKP (Polskie Koleje Państwowe) Polish Railways. [9]. The general requirements ( $\$ 72)$ of this chapter state that the primary sources of power supply for station RTCS systems are power grids that meet the requirements for:

- regulations for the construction of power equipment,

- principles of protection against overvoltage,

- principles of shock protection.

The block diagram of power supply system's general structure intended for a RTCS computer station is shown in Figure 1 [7, 9, 10, 11, 12].

The use of relay and computer based systems or computer based systems requires the provision of adequate reliability and quality of power [9]. Therefore, new, more suitable power supply systems are introduced, so that they can meet the increased requirements.

\subsection{ARS controller}

Due to the above, based on the analysis of the requirements and technical solution of the currently produced power supply systems of station RTCSs a development of an integrated ARS controller was proposed. This controller integrates power components and contains:

- measurement systems,

- logic of the ARS system operation,

- diagnostics system with data transfer interfaces.

The measuring system checks the correctness of the power supply of $230 / 400 \mathrm{~V} \mathrm{AC}+/-10 \%$. If the measured voltage is not within the given measurement range, then a logic signal is generated to the controller's system logic block. Based on logic rules and set priorities, this system turns on or off relays that turn on voltage for station equipment. Information about the status of the power elements is transferred to the control panel and the remote diagnostics system. The location of this controller in the power supply system is shown in Fig. 2.

\subsection{Application steps of ARS controller}

Based on the analysis of current solutions $[2,10,11,12]$ and the requirements for power supply systems of station RTCS [9], a functional model of a controller with a Petri Net has been developed. Based on the developed net and the use of the NI LabView graphical programming language, a dedicated software application has been developed. In parallel to the design and implementation of the software, several assumptions have been made regarding the hardware platform of the controller. NI CompactRIO platform has been selected as the development platform. The next step of the ARS controller implementation is the integration of the software application developed for the hardware platform. Following the integration and positive activation of the controller, functional tests were performed on the basis of a previously developed research program. This enabled for the verification of assumed assumptions for the ARS controller.

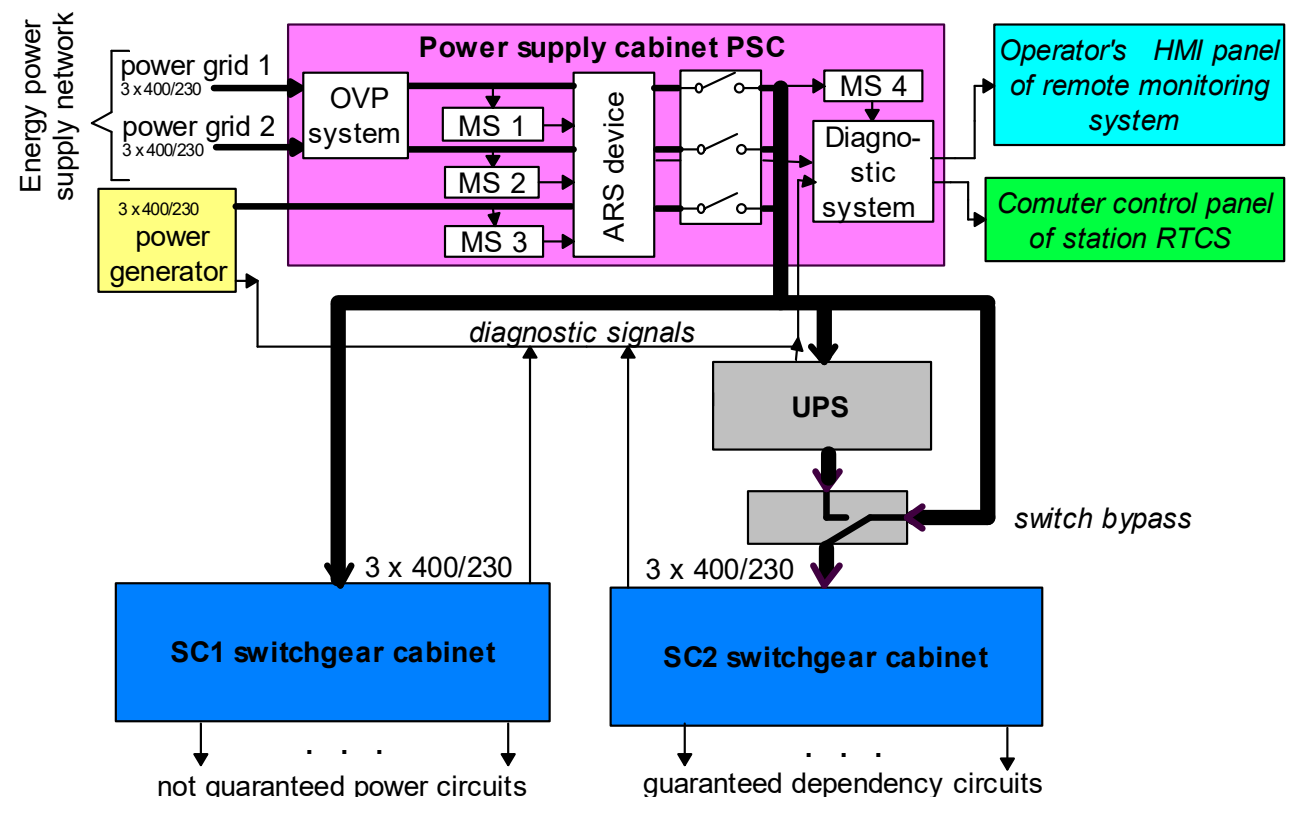

Fig. 1. General structure of a power supply system for the computer station RTCS's [7]. 


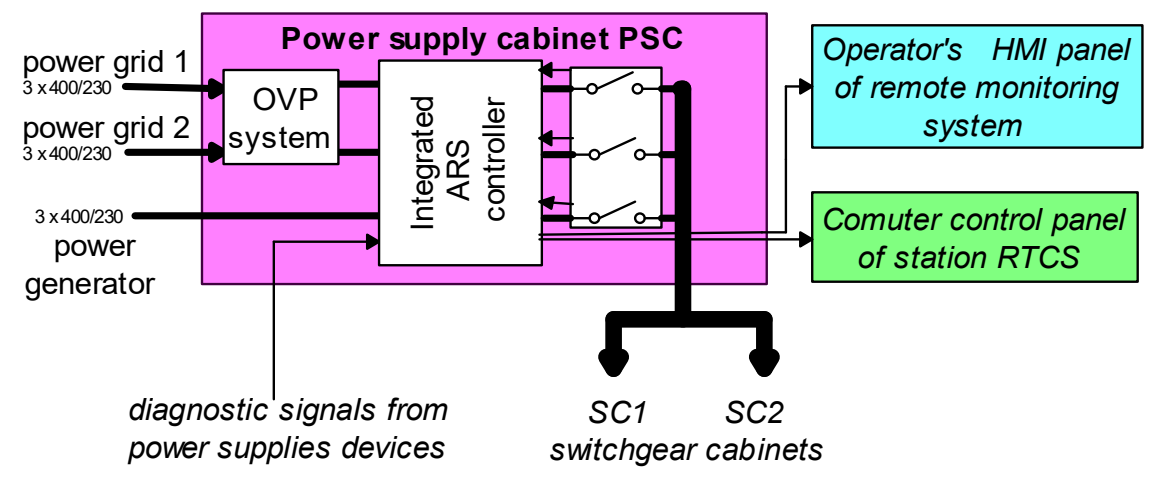

Fig. 2. Structure of the station RTCS power supply system with an ARS controller.

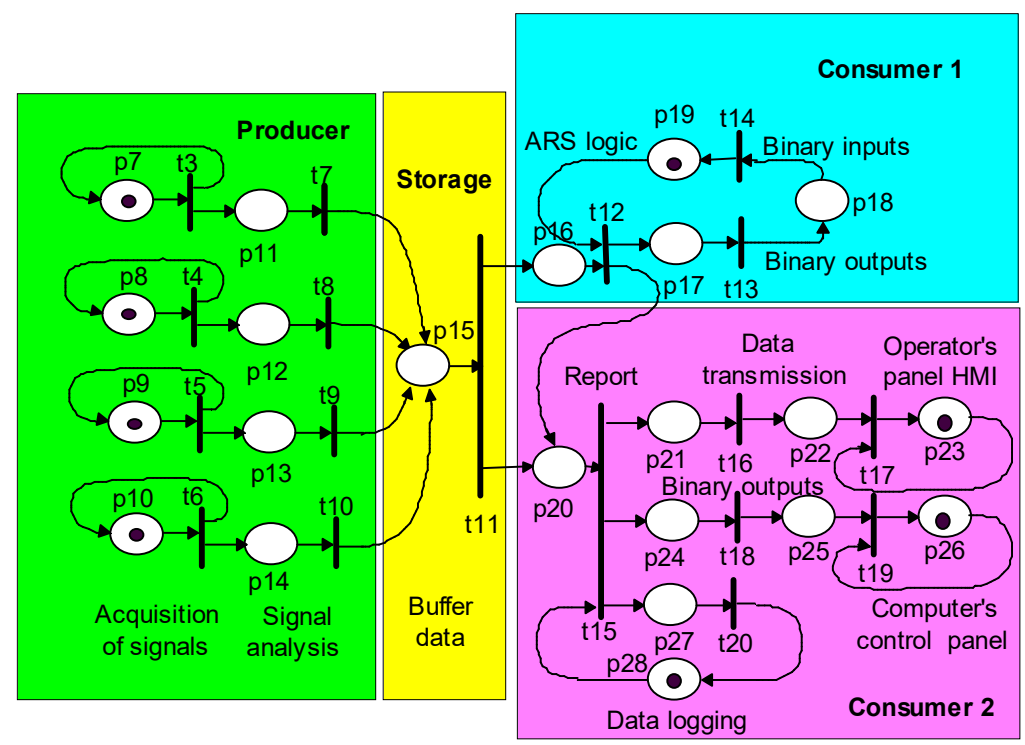

Fig. 3. The ARS controller Marked Petri Net.

\section{ARS controller's Petri Net}

\subsection{Petri Nets}

In 1962 Carl A. Petri developed based on graph theory based tool called Petri Nets (PN) to model discrete systems [13-17]. As a result of long-term development of the theory of these nets a number of classes of PNs have emerged for which numerous applications have been established [4, 5, 6, 7, 8, 13, 14]. During the development phase of assumptions and design requirements as the graphical tool for modeling of complex systems Petri Nets allow for a good and clear communication between designers and customers.

Therefore, Marked Petri Net (MPN) [15, 17]:

$$
N=\left(P, T, A, M_{0}\right)
$$

where the following conditions are met:

$N=(P, T, A)$ is the $\mathrm{PN}$,

$M_{0}: P \rightarrow \mathbb{Z}_{+}$it is a function defined on the set of places called initial markings of the $N$ net.

In this class of nets places have an unlimited capacity for tokens, but arcs can carry only one token. The execution of transition involves eliminating of individual tokens from the input places and adding the individual tokens to the exit places of this performed transition. In the class of Generalized Petri Nets $(G P N)[15,17]$ it is possible to eliminate or add to one place more tokens in a single transition by assigning appropriate weights to the arcs. By introducing the function of limiting capacity of the places for tokens to the GPN, one of the most common net classes in literature [15, 17] called Places and Transitions Petri Net (PTPN) is obtained [15, 17]. The PNPT can easily replace a GPN which has the same properties. Accordingly, the term PTPN often refers to GPN in the literature $[15,17]$. It can be stated that the MPN class is a special case of GPN and PTPN in which there is an unlimited token capacity and arcs carry only one token (the weight of the arcs is 1).

\subsection{ARS controller Marked Petri Net}

Based on the features of the ARS integrated controller power supply system of station RTCS, a Marked Petri Net (ARS controller MPN) has been developed for the ARS controller. This MPN in which the capacity of the places are unlimited and only one token can pass through the arc is a special case of the GPN and PTPN class of a PN. The developed MPN for the ARS controller can highlighted in Figure. 3. It includes 
- Producer process for generating tokens

- Storage process (token buffer)

- Two consumer processes for absorbing tokens.

Figure 4 shows the hierarchical simplified MPN for the ARS controller. This net was developed as a result of the hierarchy of the ARS controller MPN from Figure. 3, using the bottom-up method of creating [17]. The resulting hierarchical simplified MPN is easier to analyze. This net corresponds to the producer-consumer model system. Classes of this type net are well known in literature [17]. One of the important features of this net is its liveliness. This means that the net does not get jammed, and every producer's token is consumed by consumer processes [17]. Fig. 5 shows the blockdiagram of the ARS controller with the macro transition MT1 from the hierarchical simplified Marked Petri Net.

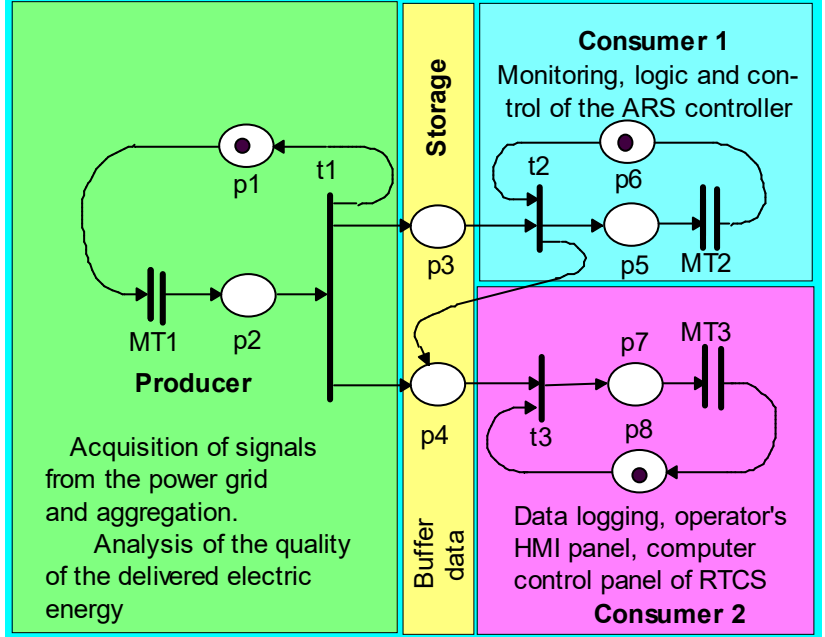

Fig. 4. The ARS controller hierarchical simplified Marked Petri Net [7].

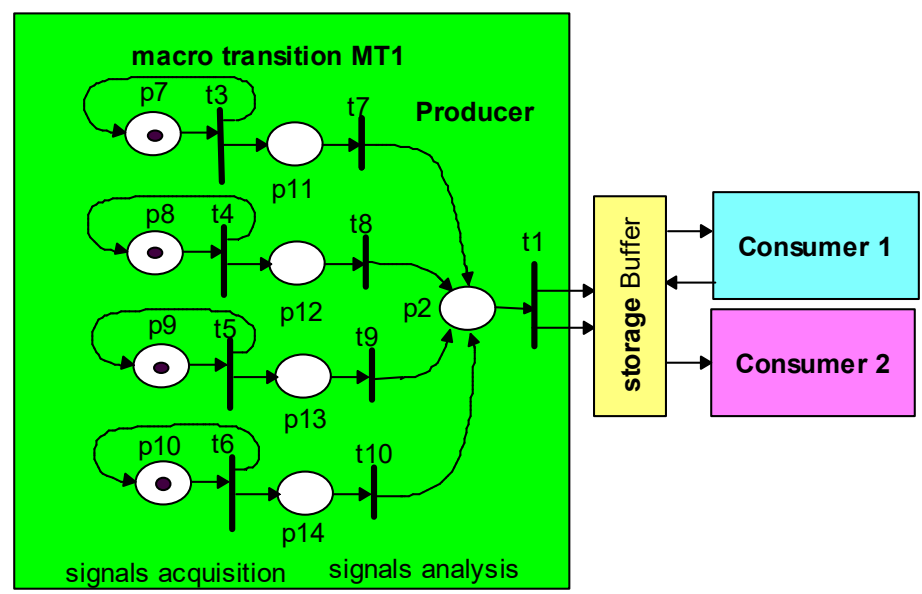

Fig. 5. The ARS controller block'-diagram with the macro transition MT1.

\section{Practical implementation of the ARS controller}

\subsection{Hardware platform of ARS controller}

The ARS controller hardware platform was configured using NI CompactRIO components [8]. It is a modular real-time platform. It meets the stringent requirements of environmental standards approved by the Management of the Polish Railway Lines. This platform consists of:

- NI cDAQ-9135 cassette with processor module equipped with: Atom processor $1.33 \mathrm{GHz}, 32 \mathrm{~GB}$ RAM, 8 slots, Linux RT real-time operating system, interface to HMI panels,

- NI PS power supply module,

- 4 pieces of analog input modules NI 9244,

- 2 pieces of digital inputs/outputs NI 9375.

\subsection{Software application of ARS controller}

The National Instruments LabView graphical software environment $[6,8,18]$ was used to implement the ARS controller software application. This environment incorporates numerous tools for a rapid software development. The main advantage of this environment is that the execution of the program is based on the data flow between the nodes of the program. These include simple functions, complex functions, VI Express functions and SubVI functions developed by users. Executing the node function will only occur if the node inputs will have data. This requirement is similar to the mechanism of triggering transitions in Petri Nets. This environment also includes programming structure patterns. One of them is the producer-consumer pattern. It transmits data from the producer to the consumer through the data buffer so that no data is lost. The pattern of this structure was used in the software application of the ARS controller. In the application the authors also used the previously mentioned mechanism of building own subprograms (SubVI). For the analysis of the measured signals Electric Power tool was used in which functional blocks are used to analyze the quality of electricity. DAQmx functions driver have also been used for the acquisition of measured signals. Software in the LabView environment is developed in two windows. The first one, called the Front Panel, is responsible for the communication between the operator and the system. In the second window, called the Block Diagram, the program code of the application is placed. 


\section{Conclusions}

Following the integration of the hardware platform and the application software as well as the launch of the ARS controller, functional laboratory tests of the controller were performed. Laboratory tests were carried out on the developed controller. Three regulated 230/400 V AC power supplies with a wide range of output voltage regulation were connected to the controller. These studies were made on the basis of a previously prepared research program. After minor modifications to the controller's application software it can be concluded that the ARS controller's operation is correct. This tested controller and the documentation were provided to a manufacturer of the power supply system of the RTCS stations. The controller is currently undergoing field testing. Once completed it will be possible to apply for its admission for the use of the integrated ARS controller in RTCS stations within the Polish Railway Network.

\section{References}

1. W. Winiecki, Organization of computer measuring systems, Warsaw University of Technology Publishing House, Warsaw, 2006) [In Polish]

2. M. Dąbrowa-Bajon, Basics of railway traffic control (Warsaw University of Technology Publishing House, Warsaw 2002) [In Polish].

3. A. Lewiński and Perzyński, New solutions of control computers in railway traffic control systems on the example of SSP, (Conference materials, Transport in the 21st century, Warsaw 2001) [In Polish]

4. R. Mielnik, Petrii Net as a tool for modeling a microprocessor measurement-control system using in critical application, (Proceedings of the IMEKOTC7 Symposium, Measurement Science of the Information Era, Cracow 2002).

5. R. Mielnik, Petri Net in the design of metering and control systems for discrete events in critical applications, (Collective work edited by A. St. Jagiełły - Electrical engineering in traction applications, Monograph no. 450, Cracow University of Technology 2014) [In Polish]
6. R. Mielnik, Synthesis of controller for railway level crossing devices using Petri Nets and state machine, (Technical Transactions, Electrical Engineering, Issue 1-E (2), Cracow University of Technology, 2016).

7. R. Mielnik, Synthesis of control devices of ARS power suplly railway network usig Petri nets and LabView, (Proceedings the XVII Conference Electric Traction, Semtrak'2016, Zakopane 2016, Poland) [In Polish]

8. National Instruments website www.ni.com, 2017

9. Technical guidelines for the construction of railway traffic control devices, Ie-4 (WTB-E10), (Polish Railway Lines, Warsaw, 2014) [In Polish]

10. M. Kornaszewski, Backup power supply systems for railway traffic control devices, Journal "Logistyka", Łódź, 2013) [In Polish]

11. Album of power devices: Supply system for SZUS type control devices, (KZA Kraków, 1995) [In Polish]

12. Technical guidelines for the construction of railway traffic control devices, Ie-4 (WTB-E10),, ( Polish Railway Lines, Warsaw, 2014) [In Polish]

13. David, R., and Alla, H.: „Discrete, Continuons, and Hybrid Petri Nets"' (Springer, 2005)

14. B. Hruz. and M.C. Zhou, Modeling and Control of Discrete-Event Dynamic Systems with Petri Nets and other tool, (Springer, 2007)

15. P. H. Starke, J. Żurek, Petri Nets: basics, applications, theory,. PWN, Warsaw, 1987)

16. T. Szmuc, M. Szpyrka, Formal methods in software engineering of real-time systems, WNT Warsaw 2010.) [In Polish]

17. M. Szpyrka, Petri Nets in modeling and analysis of concurrent systems, WNT, Warsaw 2008) [In Polish]

18. Z. Wróbel and R. Ziemba, Simulation of atmospheric surges in the systems of non-traction power lines supplying railway traffic control devices, (Technical Transactions, Electrical Engineering, Issue 1E/2009, nr 15, Cracow University of Technology, 2009) [In Polish]. 